\title{
9.
}

\section{Ueber Reihen von Kegelschnitten in einer Ebene, welche sich in denselben vier Puncten schneiden.}

(Von Herrn Jacobi, Königl. Preufs. Lieutenant in der sechsten Artillerie-Brigade zu Breslau.)

In der „Systematischen Entwicklung der Abhängigkeit geometrischer Gestalten von einander, von Herrn Steiner" bilden die Gerade und der Strahlenbüschel die Grundlage der Entwicklungen. Man sieht, dafs zwei projectivische Geraden zwei collineare Systeme bilden, in denen die Puncte jedes Systems in einer Geraden liegen; eben so stellen zwei projectivische Strahlenbüschel zwei collineare Systeme dar, in denen die Geraden jedes Systems durch einen und denselben Punct gehen.

So wie nun zwei schief liegende Geraden oder Strahlenbüschel, welche projectivisch sind, einen Kegelschnitt erzeugen: eben so erzeugen zwei nicht collinear liegende collineare Systeme Reihen von Kegelschnitten, welche sich in denselben drei Puncten schneiden, oder dieselben drei Geraden berühren.

Es ist kein Zweifel, dafs die Gesetze der Collineation und Reciprocität sich allgemein nach der Methode des Herrn Steiner entwickeln lassen. Besonders wichtig hierbei ist, dafs diese Geometrie durch besondre Lagen der Grundgebilde das Imaginäre der Analysis in ihr Gebiet zieht.

Legt man zwei projectivische Geraden so aufeinander, dafs die Durchschuitte der Parallelstrahlen sich decken, so bilden die entsprechenden Punctenpaare beider Geraden conjugirte Puncte der Involution. Liegen die Geraden ungleich liegend, so giebt es zwei Puncte: die doppelten Puncte der Involution, in denen entsprechende Puncte beider Geraden sich decken. Es giebt keine doppelten Puncte, wenn die Geraden gleichliegend liegen. Der Centralpunct ist der Punct, in welchem die Durchschuitte der Parallelstrahlen sich decken.

Zwei projectivisch ähnliche oder gleiche Geraden bilden immer ein Involutionssystem, wenu sie zur Deckung gelangen. 


\section{与. 1.}

Je zwei entsprechende Puncte $a$ und $a^{\prime}$ der beiden collinearen Systeme $\boldsymbol{M}$ und $\boldsymbol{M}^{\prime}$ sind die Mittelpuncte projectivischer Strahlenbüschel, deren entsprechende Strahlen entsprechende Geraden jener Systeme sind. Diese Strahlenbüschel liegen schief, wenn die Systeme nicht collinear liegen, und erzengen einen Kegelschnitt $\left(a a^{\prime}\right)$.

In den drei Situationspuncten der nicht collinear liegenden Systeme decken sich entsprechende Punctenpaare; einer dieser Puncte ist stets reell; zwei können imaginär werden; alle drei liegen nie in einer Geraden.

Die Situationspuncte sind die Mittelpuncte zweier concentrischen projectivischen Strahlenbüschel. Alle Kegelschnitte $\left(a a^{\prime}\right)$ schneiden sich in den drei Situationspuncten, weil letztere stets die Durchschnitte entsprechender Strahlen sind.

Liegen die Puncte $a, b, c, \ldots$ des einen Systems in einer Geraden, so gehen alle Kegelschnitte $\left(a a^{\prime}\right),\left(b b^{\prime}\right), \ldots$ durch einen bestimmten vierten Punct, den Durchschnitt der Geraden $a b$ und $a^{\prime} b^{\prime}$.

Im Allgemeinen enthält jeder durch zwei collineare Systeme bestimmte Kegelschnitt nur ein Paar entsprechende Puncte derselben, welche sich nicht decken, da in beiden Systemen nur entsprechende Puncte Mittelpuncte projectivischer Strahlenbüschel sind.

Je zwei entsprechende Geraden $\alpha$ und $\alpha^{\prime}$ der beiden collinearen Systeme $\boldsymbol{M}$ und $\boldsymbol{M}^{\prime}$ sind in Ansehung der entsprechenden Puncte projectivisch, liegen im Allgemeinen schief, und erzeugen einen Kegelschnitt $\left(\alpha \alpha^{\prime}\right)$.

Alle Kegelschnitte $\left(\alpha \alpha^{\prime}\right)$ sind einem Dreiecke eingeschrieben, dessen Seiten die Situations - Axen sind, da letztere alle entsprechende Geraden in entsprechenden Puncten schneiden.

Es möge noch erwähnt werden, dafs, wenn von zwei collinearen Systemen die Rede ist, dieselben nicht collinear liegend gedacht werden.

\section{S. 2.}

Zwei collineare Systeme $\boldsymbol{M}$ und $\boldsymbol{M}^{\prime}$ sind mit einem und demselben Systeme $\boldsymbol{P}$ reciprok verwandt. Hieraus ergiebt sich sogleich, aus den bekannten gegenseitigen Beziehungen der Systeme, diejenige Verwandtschaft, nach welcher einer Geraden ein Kegelschnitt entspricht; alle diese Kegelschnitte schneiden sich in drei festen Puncten, und allen Geraden, welche durch einen Punct gehen, entsprechen Kegelschnitte, welche sich in einem und demselben vierten Puncte schneiden. Es ergiebt sich nemlich Folgendes: 
A. In $\boldsymbol{P}$ entsprechen:

Jedem Puncte

Allen Puncten einer Geraden

Vier harmonischen Puncten einer Geraden

Allen Geraden, welche sich in einem

Puncte schneiden,

Dreien bestimmten Geraden

$$
\text { U. s. w. }
$$

B. In $\boldsymbol{P}$ entsprechen:

\section{Jeder Geraden}

Jedem Strahlenbüschel

Allen Strahlenbüscheln, deren Mitlelpuncte in einer Geraden liegen, Dreien bestimmten Strahlenbüscheln

$$
\text { U. s. w. }
$$

in $\boldsymbol{M}$ und $\boldsymbol{M}^{\prime}$ :

In jedem System eine Gerade (Strabl).

$\mathbf{Z}$ wei projectivische Strahlenbüschel, welche einen Kegelschnitt erzeugen.

Vier harmonische Puncte eines Kegelschnitts.

Kegelschnitte, welche durch einen bestimmten vierten Punct gehen.

Die drei Situationspuncte.

$$
\text { U. s. w. }
$$

in $\boldsymbol{M}$ und $\boldsymbol{M}^{\prime}$ :

In jedem System ein Punct.

$\boldsymbol{Z}$ wei projectivische Geraden, welche einen Kegelschnitt erzeugen.

Kegelschnitte, welche eine gemeinschaftliche vierte 'Tangente haben. Die drei Situations-Axen.

\section{3.}

Es mögen jetzt die Kegelschnitte, welche sich in denselben vier Puncten schneiden, noch aus einem andern Gesichtspunct betrachtet werden.

Sind vier Puncte gegeben, welche allen Kegelschnitten gemeinschaftlich sein sollen, so kann zu jedem fünften Puncte nur ein Kegelschnitt gefunden werden. Wir stellen uns jetzt die ganze Reihe der durch vier Puncte möglichen Kegelschnitte vor. Alsdann kann man drei beliebige jener vier Puncte als Situationspuncte und durch den vierten Punct zwei beliebige Geraden als entsprechende Geraden zweier collinearen Systeme annehmen. Dies ist möglich, weil durch diese vier Geraden beider Systeme und das Entsprechen derselben, die collinearen Systeme bestimmt sind. Die durch den vierten Punct gehenden Geraden schneiden jeden Kegelschnitt in entsprechenden Puncten beider Systeme. Man kann nun ferner alle Puncte eines Kegelschnitts als entsprechende Puncte einer Reilse von collinearen Systemen anselien. Alle diese Systeme haben dieselben drei Si- 
tuationspuncte, und alle übrigen Puncte jedes Systems liegen in einer Geraden, welche durch den gemeinschaftlichen vierten Punct der Kegelschnitte g eht. Alle diese Geraden sind unter sich projectivisch und liegen schief.

Diese Betrachtungen führen unmittelbar zu einer Reihe von Sätzen.

1. Schneiden sich vier Kegelschnitte in denselben vier Puncten, und legt man durch einen dieser Puncte zwei Geraden, welche jene Kegelschnitte schneiden, so sind die Doppelverhältnisse aus den Entfernungen der entsprechenden Durchschuittspuncte in beiden Geraden gleich.

Diese beiden Geraden schneiden die Seiten desjenigen Dreiecks, welches die andern drei gemeinschaftlichen Durchschnittspuncte der Kegelschnitte bilden, in entsprechenden Puncten.

2. Ist einem Kegelschnitte ein Dreieck eingeschrieben, und zieht man durch einen Punct des ersten zwei Gerade, welche die drei Seiten des Dreiecks und den Kegelschnitt schneiden, so erhält man in jeder Geraden vier Puncte, und die Doppelverhältnisse aus den Entfernungen dieser vier Puncte in beiden Geraden sind einander gleich.

Hierin liegt offenbar die Construction eines Kegelschnitts aus fünf gegebenen Puncten. Man beachte dabei nur, dafs die in einer Seite des Dreiecks liegenden Puncte beider Geraden entsprechende Puncte sind.

3. Ist einem Kegelschnitte ein Dreieck eingeschriebeu, und drehen sich zwei Gerade, welche sich stets im Kegelschnitte schneiden, um zwei feste Puncte einer Seite des Dreiecks, so sind die Doppelverhältnisse aus den Entfernungen der jedesmaligen Durchschnittspuncte dieser Geraden mit dem Kegelschnitte von den beiden andern Seiten des Dreiecks, constant; und zwar gleich dem Doppelverhältnisse aus den Entfernungen der beiden festen Puncte von jenen Seiten des Dreiecks.

4. Ist einem Kegelschnitte ein Viereck eingeschrieben, so ist das Doppelverhältnifs aus den Entfernungen jedes Punctes des Kegelschnitts voll den vier Seiten des Vierecks, constant.

Diesen Beziehungen liegen die metrischen Relationen collinearer Systeme zum Grunde.

5. Schneiden sich eine Reihe von Kegelschnitten in denselben vier Puncten, und zieht man durch einen dieser Puncte zwei Geraden, welche jene Kegelschnitte schneidern, so berühren diese Geraden und alle durch sie bestimmten Sehnen der Kegelschnitte einen und denselben Kegelschnitt. Die drei Situations-Axen sind ebenfalls Tangenten des Kegelschnitis. 
6. Sind zwei Dreiecke einem Kegelschnitte eingeschrieben, so berühren die Seiten dieser Dreiecke einen neuen Kegelschnitt.

7. Die Eckpuncte zweier einem Kegelschnitte umschriebenen Ireiecke liegen in einem Kegelschnitt. Oder:

8. Je vier Tangenten eines Kegelschnitts werden von jeder fünften Tangente projectivisch geschnitten.

\section{S. 4.}

Bei den hier angestellten Betrachtungen wird die Ansicht zum Gronde gelegt, dafs zwei oder mehrere Kegelschnitte, welche in einem Puncte eine einfache oder höhere Osculation haben, an dieser Stelle zwei oder mehrere nächst anliegende Puncte gemein haben.

Haben eine Reihe von Kegelschnitten zwei Puncte gemeinschaftlich, und berühren sie sich in einem und demselben dritten Puncte, so sind diese drei Puncle die Situationspuncte collinearer Systeme. Je zwei durch den Berührungspunct gehende Geraden liegen perspectivisch und werden von der gemeinschaftlichen Sehne aller Kegelschnitte in entsprechenden Puncten geschnitten.

9. Berühren sich eine Reihe von Kegelschnitten in einem Puncte, und schmeiden sie sich in zwei andern Puncten, so gehen alle von zwei durch den Berührungspunet gezogenen Geraden bestimmten Sehnen der Kegelschnitte durch einen Punct der gemeinschaftlichen Sehne.

Bei einer Reilıe von Kegelschnitten, welche sich in denselben beiden Puncten berühren, sind die beiden Tangenten in diesen Puncten und die gemeinschaftliche Sehne die drei Situations-Axen. Legt man durch einen Berührungspunct zwei beliebige Geraden, so liegen dieselben perspectivisch und werden von der Tangente im andere Berührungspuncte in entsprechenden Puneten geschnitten.

10. Wenn eine Reihe von Kegelschnitten sich in denselben beiden Puncten berühren, und man zieht durch einen Berührungspunct zwei die Kegelschnitte schneidende Geraden, so gehen alle durch diese Geraden bestimmten Sehnen der Kegelschnitte durch einen Punct der Tangente im zweiten Berührungspuncte. Aus der im Anfange angeführten Bemerkung folgt zunächst:

11. Wenn eine Reihe von Kegelschnitten sich in denselben beiden Puncten berühren, und man zieht durch einen Berührungspunct eine die 
Kegelschnitte schneidende Gerade, so gehen alle Tangenten der Kegelschnitte, deren Berührungspuncte in dieser Geraden liegen, durch einen Punct der gemeinsebafiliehen Tangente im zweiten Berührungspuncte.

Zieht man durch jeden der beiden Berührungspuncte einer Reihe von Kegelschnitten eine Gerade, so bestimmen alle Tangenten derselben, deren Berülırungspuncte in diesen Geraden liegen, mit den beiden gemeinschaftlichen Tangenten aller Kegelschnitte die diesen letztern umschriebenen Vierecke. Diese Vierecke haben zwei gegenüberliegende Ecken und zwei gegenüberliegende Seiten gemeinschaftlich. Die Eigenschaft der den Kegelschnitten umschriebenen Vierecke giebt Folgendes.

12. Wenn eine Reihe von Kegelschnitten sich in denselben beiden Puncten berühren, und man legt durch diese beiden Puucte zwei Geraden, welche die Kegelschnitte schueiden, so schneiden sich die durch letztere Geraden bestimmten Sehnen derselben in einem Puncte der gemeinschaftlichen Sehne aller Kegelschnitte.

Wenn eine Reihe von Kegelschnitten sich in einem Puncte dreipunctig osculiren und in einem zweiten Puncle schneiden, so enthält dieser letztere Punct einen und der Osculationspunct zwei Situationspuncte, und es folgt daraus, dals

13. Bei einer Reihe von Kegelschnitten, welche sich in einem Puncte dreipunctig osculiren und in einem zweiten Puncte schneiden, alle durch zwei durch den Osculationspunct gezogene Geraden bestimmten Sehnen der Kegelschnitte durch einen Punct der gemeinschaftlichen Sehne aller Kegelschuitte gehen.

14. Zieht man durch den Osculationspunct einer Reilhe sich in demselben Puncte vierpunctig osculirenden Kegelschnitte zwei dieselben schneidende Geraden, so gehen die hierdurch bestimnten Sehnen der Kegelschuitte durch einen Punct der gemeinschaftlichen Tangente in Osculationspunct.

Die hier angeführten Sätze, so wie die an dieselben sich knüpfenden Constructionen, sind in den ${ }$ Analytisch-geometrischen Entwicklungen von Herrn Plücker, Band I. s. 8." enthalten.

Es liefsen sich hier noch viele Sätze aufstellen. Die hier gegebenen Beziehungen zeigen, in wie fern solche zwei Kegelschnitte als collineare und collinear-liegende Systeme angesehn werden können. 
5. 5 .

Bringen wir die in \$. 3. aufgestellten Reihen von collinearen Systemen mit einem reciproken Systeme in Verbindung, so sehen wir, dafs in selbigem allen Puncten eines Kegelschnitts eine einzige Gerade entspricht. Die allen Kegelschnitten entsprechenden Geraden schneiden sich in einem und demselben Puncte, weil alle Puncte eines Systems in einer Geraden liegen.

15. Sind eine Reihe von Kegelschnitten einem Vierecke umschrieben, so gehen die Polaren jedes beliebigen Punctes dieser Kegelschnitte durch einen und denselben Punct, und alle Puncte eines Kegelschnitts haben eine einzige Polare.

Aus den metrischen Relationen reciproker Systeme ergiebt sich, dals jede die Kegelschnitte schneidende Transversale zwei sich deckende projectivische Geraden enthält; und zwar sind die in einem Kegelschnitte liegenden Puncte entsprechende Puncte. Je zwei solcher Puncte enthalten zwei Paare entsprechenden Puncte der Geraden; welches der allgemeine Character der Involution ist.

16. Eine Reihe von Kegelschnitten, welche einem Viereck umschrieben sind, werden von jeder beliebigen Transversale in Puncten geschnitten, welche ein Involutionssystem bilden. Die in einem Kegelschnitt liegenden Puncte sind conjugirte Puncte.

Jedes Seitenpaar des Vierecks, d. h., je zwei Gerade, welche die vier Puncte desselben verbinden, kann als ein Kegelschnitt angesehen werden, und es ergeben sich hieraus mehrere bekannte Sätze.

$\mathbf{Z}$ wei sich deckende projectivisch ähnliche oder gleiche Geraden bilden immer ein Involutionssystem. Diese Bemerkung führt zu folgenden Beziehungen.

17. Schneiden sich drei Kegelschnitte in denselben vier Puncten, und werden dieselben von einer Transversale so geschnitten, dafs die in letzterer sich deckenden Geraden projectivisch ähnlich sind, so schneiden je drei andre Kegelschuitte, welche durch dieselben vier Puncte gehen, diese Transversale ebenfalls ähulich.

18. Werden zwei einem Vierecke umschriebene Kegelschnitte von einer Transversale so geschnitten, dafs die Abschnitte derselben zwischen beiden Kegelschnitten gleich sind, so besteht diese Gleichheit der Abschnitte 
in derselben Transversale auch für je zwei andre dem Vierecke umschriebenen Kegelschnitte.

In diesem letztern Falle decken sich alle Mittelpuncte der Entfernungen conjugirter Puncte. Dies kann woch besonders aus folgenden allgemeinen Gleichungen der Involution bewiesen werden, in welchen $\boldsymbol{b}$ und $\boldsymbol{b}^{\prime}$ conjugirte Puncte, und $\beta$ der Halbirungspunct von $b b^{\prime}$ ist, n. s. w.; nemlich:

$$
\begin{aligned}
& m b \cdot m b^{\prime} \cdot \delta \mu=m d \cdot m d^{\prime} \cdot \beta \mu, \\
& m b \cdot m b^{\prime} \cdot \varepsilon \mu=m e \cdot m e^{\prime} \cdot \beta \mu, \\
& m d \cdot m d^{\prime} \cdot \varepsilon \mu=m e \cdot m e^{\prime} \cdot \delta \mu,
\end{aligned}
$$

wenn man $\beta \mu=0$ setzt.

Berührt eine Transversale eiuen Kegelschnitt, so ist dieser Berührungspunct ein doppelter Punct. Die doppelten Puncte sind immer paarweise vorhanden.

19. Durch vier Puncte lassen sich im Allgemeinen zwei Kegelschnitle legen, welche eine gegebene Gerade berühren.

Die Theorie der Involution giebt ferner ein Mittel an die Hand, zu entscheiden, ob durch vier Puncte ein Kegelschnitt gelegt werden kanu, der eine gegebene Gerade berührt. Man verlängere nemlich die drei Seitenpare des Vierecks, bis sie die Gerade schneiden. Diese sechs Puncte bilden ein Involutionssystem. Liegen die in der Geraden sich deckenden projectivisehen Geraden ungleichliegend, so giebt es zwei doppelte Puncte und es sind zwei Kegelschnitte möglich; liegen jene Geraden aber gleichliegend, so ist im Allgemeinen kein Kegelschnitt möglich. Im letztern Falle mufs man noch untersuchen, ob die Geraden ähnlich sind, d. h. ob der Centralpunçt in unendlicher Entfernung liegt. Sind die Geraden gleich und gleichliegend, so ist nur ein Kegelschnitt möglich, und dieser ist eine Hyperbel.

Aus der Betrachtung der doppelten Puncte folgt noch unmittelbar Nachstehendes.

20. Ist ein Viereck einem Kegelschnitte eingeschrieben, und zieht man durch einen aufserhalb des letztern liegenden Durchschnitt eines Seitenpaares des Vierecks eine Tangente an denselben, so schneidet diese Tangente jedes andre Seitenpaar in Bezug auf die doppelten Puncte harmonisch.

Dieser Satz führt zu der bekannten Construction einer Tangente eines Kegelschnittes. 
21. Ist eine Reihe von Kegelschnitten einem Vierecke umschrieben, und legt man von dem Durchschnitt eines Seitenpaares des letztern Tangenten an dieselben, so liegen sämmtliche Berührungspuncte dieser Tangenten in einer Geraden, welche die beiden andern Durchschnitte der Seitenpaare jenes Vierecks verbindet.

\$. 6.

Man kann, wenn in einer Ebene vier Puncte gegeben sind, drei dieser Puncte als Situationspuncte und durch den vierten Punct eine beliebige Gerade als Collineations - Axe eines der collinearen Systeme annehmen. In den Collineations-Axen sind die unendlich entfernten Puncte entsprechende Puncte der Systeme. Eine Hyperbel, welche durch jene vier Puncte und den unendlich entfernten Punct jener Collineations-Axe geht, bestimmt die Collineations-Axe des zweiten Systems.

22. Durch jede vier Puncte einer Ebene lassen sich unzählig viele Hyperbeln legen.

Die Asymptoten berühren die Hyperbeln in den unendlich entfernten Puncten. In einer Transversale, deren Puncte ein Involutionssystem bilden, giebt es nur dann unendlich entfernte doppelte Puncte, wenn die in derselben sich deckenden Geraden projcctivisch ähnlich oder gleich sind.

23. In jedem beliebigen Viereck kann nach jeder gegebenen Richtung eine Transversale gezogen werden, welche die Seitenpaare des Vierecks in entsprechenden Puncten $a$ und $a^{\prime}, b$ und $b^{\prime}, c$ und $c^{\prime}$ schneidet, so dals im Allgemeinen

$$
\frac{a b}{a^{\prime} b^{\prime}}=\frac{a c}{a^{\prime} c^{\prime}}=\frac{b c}{b^{\prime} c^{\prime}}
$$

ist. Diese Transversale ist die Asymptote einer Hyperbel, welche durch die vier Puncte des Vierecks geht.

Jede einer Asymptote parallele Transversale schneidet die Hyperbel im Centralpuncte der Involution.

Es ist also jede Hyperbel durch vier Puncte und die Richtung einer ihrer Asymptoten gegeben, und es findet sich ein fünfter Punct als Centralpunct der Involution dieser Richtung.

Rückt eine Transversale parallel mit sich selbst fort und schneidet die sechs Seiten eines gegebenen Vierecks, so beschreibt der Centralpunct 
der Involution dieser Transversale eine Hyperbel, welche jenem Viereck umschrieben ist.

$\mathbf{Z}_{\text {wei }}$ collineare Systeme sind vollständig bestimmt, wenn die drei Situationspuncte (oder Situations-Axen) und eine Collineations-Axe gegeben sind und der Punct bestimmt wird, in welchem die CollineationsAxen beider Systeme sich schneiden sollen.

\section{\$. 7.}

Die vier Puncte einer Ebene können zwei von einander wesentlich verschiedene gegenseitige Lagen haben, nemlich:

1) Ein Punct liegt innerhalb des von den drei andern Puncten gebildeten Dreiecks. Fin solches Viereck werde durch $\boldsymbol{A}$ bezeichnet.

2) Jeder Punct liegt aufserhalb des von den drei andern Puncten gebildeten Dreiecks. Dieses Viereck heifse $B$.

In einem Viereck $\boldsymbol{A}$ liegen die Durchschnitte der Seitenpaare desselben in den Seiten des von den äufsern Puncten gebildeten Dreiecks. Jeder Kegelschnitt, welcher dem Viereck umschrieben ist, wird von einer Seite des eingeschriebenen Dreiecks geschnitten (unter eingeschriebenes Dreieck dasjenige verstanden, dessen Eckpuncte die Durchschnitte der Seitenpaare sind). Die beiden Durchschnitte des Kegelschnitts mit dieser Seite sind zwei zugeordnete harmonische Puncte zu den beiden in derselben Seite liegenden Eckpuncten des Dreiecks. Die Lage der harmonischen Puncte in einer Geraden zeigt, dafs kein Kegelschnitt dem Vierecke umschrieben werden kann, so dafs alle drei Durchschnitte der Seitenpaare desselben innerhalb dieses Kegelschnittes liegen. Zieht man nun von dem aufserhalb des Kegelschnitts liegenden Durchschnitt eines Seitenpaares Tangenten an denselben, so sieht man, dafs die vier Puncte des Vierecks auf verschiedenen Seiten der Tangenten liegen. Hieraus folgt sogleich Nachstehendes.

24. Um ein Viereck $A$ lassen sich uur Hyperbeln beschreiben.

Jede beliebige vier Puncte einer Parabel bilden nothwendig ein Viereck $B$. Es lassen sich aber auch un dieses Viereck beliebig viele Hyperbeln legen. Eine unendich entfernte Transversale schneidet diese Hyperbeln in Puncten, welche ein Involutionssystem bilden. Diese Transversale berührt die Parabel, und in dem Berührungspüncte liegt ein doppelter Punct der Involution; letztere sind aber immer paarweise vorhanden. 
25. Durch jede vier Puncte einer Parabel lärst sich eine zweite Parabel beschreiben.

Hieraus folgern wir, da äber die vier in einer Parabel gegebenen Puncte keine weitere Bestimmung gemacht werden kann, dafs sich

26. Um ein Viereck $B$ zwei, aber auch nur zwei Parabeln legen lassen.

Durch die Seiten eines Vierecks $B$ und die beiden um dasselbe beschriebenen Parabeln wird die Ebene in mehrere Theile zerlegt. Betrachten wir nun einen fünften Punct, so kann derselbe folgende Lagen haben:

1) Innerhalb des Vierecks;

2) In den Räumen der Scheitelwinkel des gegebenen Vierecks.

In beiden Lagen lassen sich durch die fünf Puncte nur Hyperbeln legen, weil vier dieser Puncte stets ein Vierek $\boldsymbol{A}$ bilden.

3) Der fünfte Punct liegt innerhalb beider Parabeln, aber aufserhalb des Vierecks.

4) Der fünfte Punct liegt aufserhalb beider Parabeln, aber nicht in den Räumen der Scheitelwinkel des Vierecks.

In beiden Fällen ist durch diese fünf Puncte nur eine Hyperbel möglich. Man lege durch den fünften Punct eine Transversale und bestimme in derselben den conjugirten Panct dieses fünften Punctes, welcher in demselben Kegelschnitte liegt. Giebt man der Transversale durch jenen Punct beliebige Lagen, so sieht man, wie die Räume 3) und 4) miteinander correspondiren. Die Räume 4) erstrecken sich ins Unendliche; es wird also auch der conjugirte Punct des gegebenen fünften Punctes durch unendliche Puncte gehen, wenn die Transversale sich um den gegebenen Punct dreht. Der Kegelschnitt ist eine Hyperbel.

In den Fällen 1) und 2) liegen die Puncte der Hyperbel ebenfalls in beiden Räumen 1) und 2).

5) Der fünfte Punct liegt innerhalb der einen und aufserhalb der andern Parabel.

Lärst man eine Transversale um diesen fünften Punct sich drehen, so sieht man, dafs sein conjugirter Punct in den durch den Fall 5) gegebenen vier Räumen fortrückt. Alle diese conjugirten Puncte liegen aber nothwendig in endlicher Entfernung, da es in diesen Räumen nur zwei unendlich entfernte Puncte giebt, welche aber in den Parabeln selbst liegen. 
27. Um ein Viereck $B$ lassen sich Hyperbeln, Ellipsen und zwei Parabeln beschreiben. Alle Puncte der Ellipsen liegen innerhalb einer und auferhalb der andern Parabel. Alle Puncte der Hyperbeln liegen innerhalb oder aufserhalb beider Parabeln.

Aus den aufgestellten Sätzen folgt, dafs, wenn eine Transversale sich nach einer von zwei bestimmten Richtungen parallel fortbewegt und ein Viereck $\boldsymbol{B}$ schneidet, der Centralpunct der Involution in beiden Fällen eine Parabel beschreibt.

Es möge hier abgebrochen werden. Es ist leicht zu sehen, dafo noch eine grofse Reihe von Sätzen und Constructionen entwickelt werden könnten und dafs die Verwandtschaft der Collineation selbst noch einige neue Entwicklungen gestatten würde.

Die aufgestellten Sätze und Benennungen sind aus allgemein bekannten Werken entlehnt.

Breslau, den 19. Angust 1841. 